CRITICA, Revisla Hispanoemericana de Fillosoffa

Vol. XX, No. 69 (agosto 1988): 79-107

CONTINGENCIA A PRIORI

MARgarita M. VALDÉS

Instituto de Investigaciones Filosóficas, UNAM

\title{
I. Introducción
}

En su trabajo intitulado Naming and Necessity, ${ }^{1}$ Saul Kripke pretende introducir la posibilidad de conocer $a$ prior $^{2}$ verdades contingentes, esto es, proposiciones que resultan ser verdaderas del mundo real, $m^{*}$, pero que podrían ser falsas respecto a algún otro mundo posible $m_{n}(N \& N$, pp. 54-55). La tesis es presentada como una consecuencia de otras tesis centrales en la obra de Kripke: (1) El carácter necesario o contingente de las proposiciones es independiente de nuestro conocimiento de ellas; la categoría de necesidad pertenece al ámbito de la metafísica, en tanto que la de aprioridad al de la epistemología; éstas son categorías lógicamente independientes y no hay argumentos convincentes que las conecten, esto es, que demuestren que una proposición $p$

1 Saul A. Kripke, Naming and Neceserily (en adelante se citará $N \& N$ ), Basil Blackwell, Oxford, 1980 . Edición revisada y aumentada. (Traducción al castellano, $E l$ nombrar y la neceridad, UNAM, México, 1985.)

2 En la nota 26 de $N \& N$, Kripke 'estipula' que llamará 'a priori' a los enunciados cuya verdad se siga de una 'definición' que fija la referencia de un nombre. En otros pasajes, en cambio, utiliza la expresión "a priori" con una acepción más amplia para calificar a aquellos conocimientos que obtenemos sin necesidad de mirar cómo es este mundo. Es en este segundo sentido, bastante vago por otra parte, que entenderemos la expresión "a priori" en este trabajo. De otra manera la verdad de la tesis de Kripke se seguirfa trivialmente de su propia estipulación. 
es necesaria si, y sólo si, $p$ es conocida a priori; (2) los nombres propios son designadores rígidos, es decir, si designan a un individuo $x$ en $m^{*}$ designarán a ese mismo individuo en todo $m_{n}$ en el que exista $x$; las descripciones definidas, en cambio, no son designadores rígidos, ${ }^{3}$ pues es posible que designen a un individuo $x$ en $m^{*}$ y a otros objetos diferentes de $x$ en otros mundos posibles. "Así, por ejemplo, el nombre propio "Aristóteles". designa en todo mundo posible o situación contrafáctica al mismo individuo al que de hecho designa este nombre en el mundo real, en tanto que la descripción "el discípulo más notable de Platón" designa a Aristóteles en $m^{*}$ pero podrá designar a otros individuos diferentes de Aristóteles en otros mundos posibles, por ejemplo, en un mundo posible en el que Aristóteles muere a los cinco años. (3) Una descripción definida usada para identificar o seleccionar al individuo que haya de ser el referente de un nombre, fija simplemente la referencia del nombre en cuestión, pero no nos proporciona un sentido necesariamente asociado al nombre, ni convierte al

3 Kripke distingue, como es bien sabido, entre descripciones definidas esenciales y accidentales. Las primeras son designadores rígidos debido a que seleccionan a su referente por ser el único individuo que posee cierta propiedad esencial, la cual, desde luego, será tenida por el objeto en todo mundo posible en el que exista. Las descripciones definidas accidentales, en cambio, son designadores no rigidos, pues seleccionan un único objeto mediante una propiedad accidental. A lo largo de este trabajo, cuando se hable de "descripciones" ha de entenderse que se trata de descripciones definidas accidentales. Esta clase de descripciones son las comúnmente usadas para fijar la referencia de un nombre y las que se requieren para que surja la posibilidad de conocer a priori verdades contingentes. No trataré en este trabajo la tesis kripkeana conforme a la cual habría también verdades necesarias conocidas a posteriori.

M. Dummett en su libro Frege. Philosophy of Language, Duckworth, Londres, segunda edición, 1981, esp. pp. 112-115, alega que la distinción entre designadores ŕgidos y no rf́idos se reduce en último término a la distinción entre términos singulares con alcance amplio y restringido respectivamente en contextos modales. Kripke rechaza esta interpretación en la “Introducción” a $N \& N$, esp. pp. 5-9 y 14. 
nombre en una especie de abreviatura de la descripción; la relación entre el nombre y la descripción accidental que fija su referencia es contingente. Esto explica el hecho de que cuando fijo la designación de un nombre, pongamos como ejemplo "Kripke", mediante una descripción definida, digamos "el autor de $N \& N$ ", puedo más tarde afirmar sin contradecirme que Kripke podría no haber sido el autor de $N \& N$; "el autor de $N \& N$ " sólo se usó en $m$ " para fijar la referencia de "Kripke" en todo $m_{n}$, pero no para establecer una identidad de sentidos entre la descripción y el nombre. La rigidez del nombre y la no rigidez de la descripción hacen imposible sostener que el nombre y la descripción que fija su referencia "signifiquen" lo mismo, o hayan de designar, necesariamente, al mismo individuo en toda situación contrafáctica o mundo posible.

A las tres tesis anteriores podríamos añadir una cuarta tesis kripkeana, la de que el nombre no tiene, en contra de lo que pensó Frege, un sentido o un significado descriptivo necesariamente asociado. Conocemos directamente a los individuos en nuestras inmediaciones (y no sólo indirectamente a través de sus rasgos cualitativos) y les damos nombres que refieren directamente a ellos (y no indirectamente a través de predicados satisfechos únicamente por el referente del nombre). Decir que los nombres no tienen un sentido fregeano quiere decir, de acuerdo con Kripke, dos cosas: (a) que no tienen un significado conceptual o descriptivo asociado necesariamente y (b) que en el caso en que asociemos mentalmente alguna descripción a un nombre, no será esa descripción la que determine la referencia del nombre $(N \& N$, p. 59$)$. No son las descripciones que usamos eventualmente para fijar la referencia de un nombre las que determinan su referencia; ésta se determina, en el caso de la introducción de un nuevo nombre, mediante la 
intención del hablante de referirse a un individuo particular $y$, en el caso en que algún hablante use un nombre previamente introducido en el lenguaje, por la cadena causal que va desde el uso de ese hablante en esa ocasión particular hasta el bautismo originario en el que alguien le asignó un referente al nombre en cuestión.

De esta manera, si aceptamos las tesis anteriores parece seguirse, nos dice Kripke, la consecuencia de que al menos en algunas ocasiones es posible conocer a priori ciertas verdades contingentes expresadas mediante identidades que hacen uso de un nombre y de una descripción definida accidental. Veamos cómo se deriva dicha supuesta consecuencia siguiendo para ello un ejemplo del propio Kripke. Consideremos el caso en el que, antes de haber observado a Neptuno, Leverrier introduce el nombre "Neptuno" ("N" de aquí en adelante) para designar a cualquiera que sea el objeto que satisfaga la descripción "la causa de las perturbaciones en la órbita de Urano" ("el $\varphi$ " de aquí en adelante). Parece claro que al introducir Leverrier " $N$ " de esta manera, no está usando ni proponiendo que se use "el $\varphi$ " como una abreviatura o un sinónimo de "N". Si así fuera, Leverrier no podría afirmar sin contradecirse que $\mathrm{N}$ podría no haber sido el $\varphi$, y su afirmación de que $\mathrm{N}$ es el $\varphi$ sería trivial o analítica. El hecho de que Leverrier pueda pensar consistentemente en una situación contrafáctica en la que debido, por ejemplo, a un accidente cósmico $\mathrm{N}$ no fuese el $\varphi$, muestra po: lo menos dos cosas: (1) que la proposición de que $\mathrm{N}$ es el $\varphi$ es contingente, pues su negación es posible, y (2) que el significado o sentido de "el $\varphi$ " no es el significado o sentido (si es que hay alguno) de "N"; "N es el $\varphi$ " es una oración sintética cuya verdad no se sigue solamente de los significados de los términos que aparecen en ella. Así, un resultado de lo anterior parece ser el de que al afirmar Leverrier 
que $\mathrm{N}$ es el $\varphi$, antes de haber observado a Neptuno por el telescopio, estaba expresando una proposición verdadera (puesto que $\mathrm{N}$ efectivamente es el $\varphi$ ), contingente (dado que es concebible que $\mathrm{N}$ no fuese el $\varphi$ ) y, dado que Leverrier fijó la referencia de " $N$ " mediante la descripción "el $\varphi$ ", una proposición cuya verdad Leverrier sabe a priori, esto es, sabe que es verdadera sin necesidad de recurrir a ninguna experiencia u observación ulterior. ( $N \& N$, pp. 56 y 79.)

La tesis de Kripke, como vemos, no se apoya sólo en algunos casos aislados de enunciados que serían contingentes si bien conocidos a priori, sino que es una tesis general $1^{5}$ conforme a la cual contamos con un mecanismo en nuestro lenguaje que nos permite la introducción sistemática de enunciados con aquellas características. Veamos, pues, cómo funcionaría dicho mecanismo.

Sea $N$ un nombre cualquiera (un designador rígido)


un objeto $x$ y mediante la cual fijamos la referencia de $N$. "El $\varphi$ " es, además, una descripción accidental de $x$, es decir, una descripción que satisface $x$ en $m^{*}$ pero que podría no satisfacer en otros $m_{n}$. Asumamos que no tenemos ningún conocimiento directo de $x$, ni ninguna otra forma de identificar a $x$ independiente de la descripción "el $\varphi$ ". Si en esas condiciones afirmamos que $N$ es el $\varphi$, nuestra afirmación parece expresar una verdad contingente cuyo conocimiento no depende de ninguna observación ulterior y, en ese sentido, es a priori. Cualquier nombre y cualquier descripción que se ajustasen al esquema anterior podrían, pues, generar proposiciones contingentes de las que el hablante tendría supuestamente un conocimiento a priori. Tomemos, por ejem-

s Cfr. K. Donnellan, "The Contingent a priori and Rigid Designation", en Contemporary Peropectives in the Philorophy of Language, P. French, T. Uehling y H. Wettstein (eds.), Univ. of Minnesota Press, 1979. 
plo, el nombre "Acautli" y la descripción "el primer azteca sacrificado en honor a Huitzilopochtli"; decimos entonces:

(E) Llamaré "Acautli" a quien sea que haya sido el primer azteca sacrificado en honor a Huitzilopochtli.

Con esto hemos fijado la referencia de "Acautli". A continuación afirmamos:

(C) Si existe (existió) el primer azteca sacrificado en honor a Huitzilopochtli, Acautli es el primer azteca sacrificado en honor a Huitzilopochtli; o, simplemente

$\left(C^{\prime}\right)$ Acautli es el primer azteca sacrificado en honor a Huitzilopochtli.

(C) expresa una proposición contingente, pues es concebible un mundo posible en el que su antecedente sea verdadero y su consecuente falso. $\left(\mathrm{C}^{\prime}\right)$ también expresa una proposición contingentemente verdadera (asumiendo, desde luego, que algún individuo satisfaga la descripción "el primer azteca sacrificado en honor a Huitzilopochtli"). Sin embargo, dada nuestra estipulación (E), parece que no tenemos que apelar a ninguna observación o experimento en el mundo para poder establecer la verdad de $(C)$ y $\left(C^{\prime}\right)$ y, en este sentido, serían verdades conocidas a priori. ${ }^{6}$

La mera posibilidad de que existan enunciados contingentes cuya verdad sea conocida apriori parece ir en contra de nuestras intuiciones y de las de toda la tradición filosófica. En efecto, por un lado, se piensa

\footnotetext{
- Para que un nombre pueda introducirse de la manera antes señalada, de acuerdo con Kripke, es necesario que se trate de un nuevo nombre. Es evidente que no podemos fijar a nuestro arbitrio la referencia de nombres que ya tienen un uso establecido en nuestra comunidad lingürstica.
} 
que si hay algo que podamos conocer a priori, es decir, sin necesidad de mirar cómo es este mundo, lo conocido habrá de ser algo verdadero de todo mundo posible. Si la manera como es este mundo no tiene que ser investigada para establecer la verdad de $p$, entonces $p$ ha de ser verdadera necesariamente. Por otro lado, si $p$ es contingente, esto es, si $p$ es verdadera de este mundo pero no de cualquier mundo posible, parece indispensable indagar mediante la observación cómo es este mundo para conocer la verdad de $p$. Kripke mismo expresa este tipo de consideraciones ( $N \& N$, pp. 38-39); sin embargo, dada su tesis concerniente a la independencia lógica de las nociones epistemológicas frente a las metafísicas, concluye que no es una tesis trivial la de que sólo porque algo haya sido conocido a priori tenga que ser necesario, como tampoco es trivial la tesis de que. una verdad necesaria tenga que ser conocida a priori.

La tesis de Kripke de que pueda haber enunciados contingentes conocidos a priori ha dado lugar a una amplia discusión en la literatura filosófica reciente. ${ }^{7} \mathrm{M}$. Dummett expresa directamente que si dicha tesis de Kripke es efectivamente una consecuencia de su teoría de los nombres propios, ella es suficiente para considerar que hay algo equivocado en los argumentos que sustentan dicha teoría. ${ }^{8} \mathrm{~K}$. Donellan, por su parte, intenta mostrar que lo que Kripke piensa que es una consecuencia de su tesis acerca de la rigidez de los nombres en realidad no lo es. G. Evans, a su vez, argumenta en contra de la explicación de Donnellan y pretende encontrar en otro rasgo del lenguaje, no en la rigidez de los nombres,

7 Cfr. K. Donnellan, op. eit.; G. Evans, "Reference and Contingency", The Moniot, 1978; Stephen Schiffer, "Naming and Knowing", en French, Uehling y Wittstein (eds.), op. eit.; M. Dummett, op. cit., “Appendix" al cap. V.

Véase M. Dummett, op. cit., p. 121. 
el origen de los enunciados contingentes a priori. ${ }^{9}$ Evans intenta disolver la aparente paradoja distinguiendo entre dos tipos de contingencia, una profunda (que tiene que ver con aquello que hace verdadero a un enunciado) y otra superficial (que depende de la manera diferente como encajan los nombres y las descripciones definidas dentro del alcance de un operador modal) y mostrando que donde Kripke creyó ver una contingencia profunda, en realidad no hay más que una contingencia superficial, la cual no tiene por qué inquietarnos.

\section{La solución de Donnellan}

En su artículo "The Contingent a priori and Rigid Designation", Donnellan introduce una útil distinción en esta discusión, a saber, la distinción entre saber que $p$ es una oración verdadera y conocer la verdad expresada por $p{ }^{10}$ En la mayoría de los casos en que sé que $p$ expresa una verdad, conozco la verdad expresada por $p$, pero bien pudiera darse el caso de que aun sabiendo que $p$ es verdadera desconociera la verdad expresada por $p$. Donnellan ofrece algunos ejemplos que ilustran esta distinción. Supongamos que en un Congreso Internacional de Matemáticas escucho al matemático $X$ demostrar ante una audiencia de especialistas el teorema $p$. Los matemáticos más capaces de juzgar sobre la corrección

- La posibilidad de contar con oraciones superficialmente contingentes conocidas a priori, depende, según Evans, de que contemos con propiedades en cuya especificación se hace referencia al mundo real; por ejemplo, la propiedad de ser de hecho (o realmente) rojo. Asi, la oración “Todo lo que es de hecho rojo es rojo" es contingente en el sentido de que hay un mundo posible $m_{n}$ en el que no todo lo que es rojo en $m^{*}$ es rojo en $m_{n}$. Sin embargo conocemos aparentemente a priori su verdad. Sobre esto volveremos más adelante.

10 Kripke reconoce esta distinción que hace Donnellan en $N \& N$, p. 69 : "por supuesto, cualquiera que sepa el uso de 'es llamado' en el idioma, sabe que si 'quarks' significa algo, entonces 'los quarks son llamados "quarks" expresa una verdad, aun cuando no sepa lo que el enunciado significa". 
de la prueba se hallan presentes y celebran que se haya probado la verdad de $p$. Sin embargo, mi falta de formación matemática me impide seguir por mí misma la prueba paso por paso, pues desconozco el significado exacto de algunos símbolos que $X$ usa en su demostración. Meses más tarde aparece publicada la prueba de $p$ en el Journal of Mathematics, lo cual confirma mi creencia de que la prueba es correcta pues sé que los editores de esa revista son sumamente meticulosos. Sobre la base de la aceptación generalizada por parte de la comunidad matemática del teorema $p$, estoy en la posición de saber que $p$ es verdadero aun cuando, según Donnellan, deşconozca la verdad expresada por $p$, esto es, desconozca (parcialmente) el significado de $p$. Otro ejemplo sería el de una persona $A$ quien escucha a otra persona $B$ decir "A Xirau yo lo llamo 'Xirau'" o "Xirau se llama 'Xirau'" y $\boldsymbol{A}$ no sabe quién o qué es Xirau. $A$ puede alegar que sabe que la oración dicha por $B$ es verdadera aunque no sepa la verdad que expresa, pues no sabe acerca de qué objeto concreto es la oración, esto es, no sabe cuál individuo forma parte de la situación en el mundo que hace verdadera a $p$. ( $A$ no podría decir que la situación que hace verdadera la afirmación de $B$ es el hecho de que $B$ llama "Xirau" a Xirau, pues si no sabe quién o qué cosa es Xirau, no sabe cuál es ese hecho. Esto es, $\boldsymbol{A}$ no puede afirmar que sabe acerca o respecto de un individuo particular que él es llamado "Xirau".)

De acuerdo con Donnellan, lo único que se sigue de la tesis de Kripke de que hay designadores rígidos en el lenguaje que pueden introducirse mediante el uso de descripciones definidas que fijan su referencia, es que podemos saber a priori que ciertas oraciones son verdaderas pero no que conozcamos la verdad expresada por dichas oraciones. Esto es, con respecto al ejemplo 
de Neptuno, Leverrier sabe a priori que "Neptuno es el $\varphi$ " es una oración verdadera, e incluso puede saber $a$ priori que es contingentemente verdadera, pero no sabe cuál es la verdad expresada por la oración, esto es, no conoce (ni a priori ni a posteriori en el ejemplo) la proposición expresada. Esto, de acuerdo con Donnellan, es tanto como afirmar que Leverrier, en el ejemplo aducido por Kripke, desconoce al menos parte del significado de la oración que profiere. ${ }^{11}$

Donnellan ofrece ciertas razones intuitivas en favor de su interpretación. Veamos esto mediante otro ejemplo. Supongamos que otro astrónomo contemporáneo a Leverrier, digamos Galle (quien de hecho observó por primera vez a Neptuno) hubiese visto a través del telescopio al planeta en cuestión, le hubiese bautizado como "Neptuno", hubiese estudiado su trayectoria y después de múltiples observaciones y cálculos hubiese concluido que Neptuno, ese planeta observado por èl, es la causa de las perturbaciones en la órbita de Urano. ¿Podemos, en algún sentido, sostener que Leverrier (antes de observar a Neptuno) y Galle (habiendo observado a Neptuno) saben lo mismo cuando afirman ambos que $\mathrm{N}$ es el $\varphi$ ? ¿Podemos afirmar que ambos conocen la misma verdad? Parece que no. Es claro que Leverrier sabe que hay algo que es el $\varphi$. Esto lo sabe a posteriori, esto es, sobre la base de una serie de observaciones y cálculos, los cuales le llevan a postular, como la única explicación posible de los datos observados, que existe un cuerpo celeste que sigue una determinada trayectoria y que es la causa de las alteraciones observadas en la órbita de Urano. Leverrier puede incluso predecir la posición exacta en la que el planeta postulado se encontrará en ciertas fechas precisas. Pero Leverrier no sabe

11 Cfr. op. cil., p. 51. 
cuál objeto en el mundo real es el $\varphi$. Galle, en cambio, sabría que ese objeto, con el que él está en relación epistemológica directa mediante la observación, es el $\varphi$.

La diferencia entre el conocimiento de Leverrier y el de Galle es que parece que lo que el primero sabe es: $:^{12,13}$

$$
\text { 1. }(\exists x)\left(\underline{\left(\begin{array}{ll}
\varphi & x
\end{array}\right)}\right.
$$

en tanto que lo que sabe Galle es:

$$
\text { 2. }(\exists x)(\underline{\varphi r}) \& x=a_{i}
$$

(en donde $a_{i}$ es un término indicador: ese $a$ ).

El conocimiento que tiene Leverrier de (1) es un conocimiento a todas luces empírico. ${ }^{14}$ Lo mismo el conocimiento que tiene Galle de (2). ¿Qué añade Leverrier a su conocimiento cuando decide darle el nombre " $\mathrm{N}$ " a aquello que satisface "el $\varphi$ "? Parece que nada sustancial. Es evidente que no sabe un dato nuevo acerca de la realidad por el mero hecho de darle un nombre a cualquiera que sea el objeto que satisface "el $\varphi$ ", sin saber cuál es ese objeto. Sólo abre la posibilidad de decir que sabe que "N es el $\varphi$ " expresa una verdad, aunque, como señala Donnellan, en algún sentido no conozca (no comprenda cabalmente) la verdad expresada, es decir, no

12 En $N \& N$, p. 79 , n. 33, Kripke afirma: "En esta etapa [cuando Leverrier no habia observado a Neptuno] valía una equivalencia material a priori entre los enunciados 'Neptuno existe' y 'Existe algún planeta que perturba la órbita de tales otros planetas en tal y cual posición'". 13 En la fórmula que sigue utilizo el subrayado, siguiendo a Evans, como un operador de unicidad.

14 Para que la afirmación de Leverrier de que $N$ es el $\varphi$ tenga siquiera valor de verdad parece necesario que "el $\varphi$ " efectivamente seleccione un objeto. Leverrier sabe que hay algo que satisface la descripción "el $\varphi^{\prime \prime}$ y esto lo sabe a posteriori. Esta es la única base sobre la que puede afirmar que sabe que $N$ es el $\varphi$. Si el conocimiento de Leverrier de que $N$ es el $\varphi$ requiere o presupone un conocimiento empirico previo, ¿en qué sentido podemos afirmar que sabe a priori que $N$ es el $\varphi$ ? Para una interpretación similar, véase S. Schiffer, op. cit., p. 62. 
sepa cuál es el hecho singular (entendido éste como el tener un individuo concreto una propiedad determinada) que hace verdadera su afirmación. El conocimiento que tiene Galle de (2) es también a posteriori, pero se distingue del de Leverrier en que además de saber que algo es el $\varphi$, sabe cuál objeto es el $\varphi$, es decir, tiene una manera directa de identificar a Neptuno independiente de la descripción "el $\varphi$ " y de otras descripciones que eventualmente Leverrier pudiera usar para hablar del planeta que produce las perturbaciones en la órbita de Urano, la cual en este caso consiste en que puede caracterizarlo como ese objeto que observa en determinada posición en la bóveda celeste en un momento dado.

De acuerdo con la posición de Donnellan tenemos, pues, lo siguiente:

(i) Antes de observar Leverrier a Neptuno, Leverrier no sabe (ni a priori ni a posteriori) que $\mathrm{N}$ es el $\varphi$. Su conocimiento se reduce a saber que algo es la causa de las perturbaciones en la órbita de Urano y que la oración "N es el $\varphi$ " expresa una proposición verdadera.

(ii) Para conocer el contenido significativo de una oración en la que aparece un nombre propio es necesario el conocimiento de re del referente del nombre en cuestión (op. cit., p. 54) y lo que determina que ese conocimiento sea de re es la existencia de una conexión causal (directa o indirecta) apropiada entre el hablante que hace uso del nombre y el referente del nombre. Por lo tanto, las oraciones (en contexto directo) en las que figura un nombre $N$ están abiertas a la sustitución de $N$ por cualquier otra expresión codesignativa y están sujetas a la generalización existencial. (Rechazo de nombres "fregeanos".) Si $N$ carece de referente, de acuerdo con Donnellan, cualquier oración $p$ en la que aparezca $N$ carecerá de valor de verdad y parcialmente de significado. $\mathrm{Si}$, por 
otro lado, un nombre $N$ se introduce mediante una descripción y resulta tener un referente del que el hablante no tiene un conocimiento de re, entonces el hablante en cuestión ignorará parcialmente las condiciones de verdad de las oraciones en las que aparezca $N$ y, por ende, su contenido significativo.

(iii) De lo anterior parece desprenderse la consecuencia de que es realmente imposible la introducción de un nombre mediante una descripción cuando el hablante carece del conocimiento de re del reférente del nombre. Esto es, un hablante no puede, a la manera como lo sugiere Kripke, introducir el nombre de un objeto del cual no tiene conocimiento directo mediante una descripción y usar posteriormente ese nombre para expresar el supuesto conocimiento de un hecho singular contingente.

(iv) Si lo anterior es correcto, entonces la única manera legítima de introducir un nuevo nombre en el lenguaje para usarlo en oraciones que expresen proposiciones singulares de las que sí tenemos conocimiento, será mediante una especie de "bautismo" en el que damos el nombre a ese objeto del que tenemos un conocimiento ostensivo, directo o de re.

En suma, las exigencias que impone Donnellan para que un nombre pueda ser usado en oraciones que expresen un conocimiento de una proposición singular por parte del hablante que introduce el nombre, hacen imposible el bautismo in absentia. Leverrier, en nuestro ejemplo, no puede propiar:, ate nombrar a Neptuno hasta no verlo. Introducir el nombre "N" mediante la descripción "el $\varphi$ " y afirmar a continuación que $N$ es el $\varphi$, sin haber observado a Neptuno, no lo coloca en la posición de conocer, no digamos ya un hecho contingente $a$ priori, sino el contenido significativo de su propia afirmación. 
La mera idea de que Leverrier desconozca el significado de la oración que asevera hace reaccionar a Dummett y, más especialmente, a Evans. ¿Cómo no va a saber Leverrier el significado de la oración " $N$ es el $\varphi$ "? ¿No hay acaso algo que Leverrier entiende cabalmente cuando hace su afirmación? Parece innegable que Leverrier entiende algo cuando afirma que $\mathrm{N}$ es el $\varphi \mathrm{y}$, en este sentido, que su afirmación no carece de significado para él. Si efectivamente resulta incorrecto afirmar que Leverrier conoce o sabe cuál es el hecho concreto en el mundo que hace verdadera la oración que profiere, en lugar de pasar como lo hace Donnellan de esto a la conclusión de que Leverrier no sabe lo que significa su afirmación, tal vez resulte más adecuado sostener que lo que Leverrier entiende (el significado de su afirmación) cuando dice que $\mathrm{N}$ es el $\varphi$ es algo distinto a la proposición (o el hecho) singular expresada por la oración. ¿Qué es, pues, lo que entiende Leverrier?

\section{Proposiciones singulares vs. contenidos cognoscitivos}

Tanto Kripke como Donnellan comparten la idea de que lo que conoce quien asevera o piensa con verdad una oración singular " $F x$ " es la proposición singular expresada por " $F x$ ", esto es, el tuplo ordenado $\langle F, x\rangle$, en donde $F$ es una propiedad monádica y $x$ un individuo concreto que satisface la propiedad $F$. La proposición que $F x$, a la vez, constituye el contenido cognoscitivo de la oración " $F x^{\text {" }}$.

Ahora bien, "lo que conoce $S$ " cuando asevera o piensa que $F x$, puede entenderse de varias maneras diferentes: (a) como el hecho en el mundo denotado por " $F x$ " que hace precisamente verdadera la oración en cuestión, (b) como la proposición expresada por " $F x$ ", esto es, el par ordenado constituido por la propiedad 
$F$ y el individuo $x$ (independientemente de la manera como se le identifique) al que se le atribuye la propiedad $F,^{15}$ y (c) como el contenido del pensamiento de $S$ cuando entiende y acepta la oración " $F x$ ", es decir, el contenido cognoscitivo de la creencia de $S$ cuando sabe que $F x$. Estas nociones no han de confundirse ni identificarse, pues, como Frege mostró, es posible que la misma proposición expresada por " $F x$ " (o el mismo hecho en el mundo denotado por " $F x$ ") sea representada mediante diferentes contenidos cognoscitivos en la mente de diferentes hablantes (o del mismo hablante en diferentes momentos) que hacen uso de la misma oración " $F x$ " para expresar una creencia o un conocimiento. Sin embargo, la tesis de Kripke acerca de la referencia como una relación puramente semántica y no epistemológica, su tesis sobre la rigidez de los nombres y su teoría causal del nombrar, parecen conducirlo a una identificación tácita de las nociones de "proposición" y "contenido cognoscitivo".

La condición de verdad exigida para todo conocimiento pone énfasis en el carácter objetivo del conocimiento al requerir que lo conocido sea un hecho en el mundo, una realidad extramental, independiente del sujeto que conoce. Así, por ejemplo, para que $S$ sepa que Aristóteles es griego, se requiere que se dé el hecho en el mundo consistente en que el individuo denotado por "Aristóteles" posea la propiedad de ser griego. La condición de creencia, por otra parte, pone de relieve el carácter proposicional del conocimiento, el cual es también objetivo en el sentido de que la proposición creída es compartida por todos aquellos sujetos que comparten el mismo conocimiento, independientemente

\footnotetext{
1s La proposición expresada por una oración se caracteriza en una semántica de mundos posibles como la función asociada a la oración que lleva de mundos posibles a valores de verdad.
} 
de las diferentes modalidades como en su mente se representen la proposición creída. Así, por ejemplo, si $S$ cree que Aristóteles es griego y $S$ cree que el que de hecho fue el discípulo más notable de Platón es griego, $S$ y $S$ creen la misma proposición. Pero, además de enfatizar el carácter proposicional del conocimiento, la condición de creencia apunta a un componente mental, "subjetivo", del conocimiento, consistente en la manera distintiva como cada sujeto concibe en su pensamiento la proposición creída. Es este último componente el que nos permite hablar de un 'contenido cognoscitivo' en la mente o el pensamiento de quien cree o sabe que $F x$ diferenciable de la proposición creída y del hecho sabido. Si bien en el ejemplo anterior es correcto decir que $S$ y $S$ creen la misma proposición, no parece correcto afirmar que el contenido del pensamiento de $S$ y $S$ es el mismo. Ahora bien, Kripke parece no tomar en cuenta la manera distintiva como los diferentes sujetos epistemológicos pueden representarse mentalmente la proposición creída, esto es, parece no considerar el componente "mental" (subjetivo) del conocimiento e identificar implícitamente la noción de 'proposición' expresada por una oración singular " $F x$ " con la noción de contenido en la mente del sujeto que entiende o sabe que $F x$. De acuerdo con Dummett y Evans, es precisamente esta identificación ilegítima entre proposición expresada y contenido cognoscitivo la que permite a Kripke llegar a la consecuencia equivocada de que es posible conocer $a$ priori verdades contingentes. A una misma proposición pueden corresponderle distintos contenidos cognoscitivos en el pensamiento de distintos hablantes y al mismo contenido cognoscitivo en la mente de dos sujetos puede 
corresponderle dos proposiciones diferentes. ${ }^{16}$ Veamos cómo es esto.

Decimos que dos oraciones $p$ y $p^{\prime}$ tienen el mismo contenido cognoscitivo para un hablante $S$, si y sólo si, lo que $S$ cree cuando entiende y acepta como verdadera a $p$ es lo mismo que lo que cree cuando entiende y acepta como verdadera a $p^{\prime}$, de manera que si $S$ entiende $p$ y $p^{\prime}$ es imposible que acepte como verdadera una de estas oraciones y rechace la otra. Si $p$ y $p^{\prime}$ tienen el mismo contenido cognoscitivo para $S$, entonces $S$ considera que lo que verificaría o falsificaría a $p$ es lo mismo que lo que verificaría o falsificaría a $p^{\prime}$. Sin embargo, $p$ y $p^{\prime}$ pueden expresar distintas proposiciones desde un punto de vista puramente semántico modal, es decir, pueden encajar de diferentes maneras dentro del alcance de un operador modal. Por ejemplo, sea $p$ la oración "Acautli era hermoso" y $p^{\prime}$ la oración "El primer azteca sacrificado en honor a Huitzilopochtli era hermoso"; resulta claro que para mí, que sólo sé acerca de Acautli que fue el primer azteca sacrificado en honor a Huitzilopochtli, las oraciones $p$ y $p^{\prime}$ tienen el mismo contenido cognoscitivo o son epistémicamente equivalentes, como diría Evans. Sin embargo, es claro que si aceptamos que los nombres son designadores rígidos ( $\mathrm{y}$, por lo tanto, que "Acautli" designa al mismo individuo en todo mundo posible, es decir, a ese azteca al que de hecho designa en el mundo real) y que, por el contrario, las descripciones son no rígidas (es decir, que "el primer azteca sacrificado en honor a Huitzilopochtli" puede designar a distintos individuos en diferentes mundos posibles o situaciones contrafácticas) $p$ y $p^{\prime}$ expresan proposiciones con diferentes características modales, pues, como señala Kripke, y acepta Evans, los nombres y las des-

16 Para la noción de 'contenido cognoscitivo' véase esp. G. Evans, op. eit., pp. 176-177. 
cripciones se comportan de distintas maneras en los contextos modales.

Que las proposiciones expresadas por $p$ y $p^{\prime}$ son modalmente diferentes, puede mostrarse de la siguiente forma: la proposición expresada por $p$ es el par ordenado $\langle H,\langle a\rangle\rangle$, en donde " $H$ " representa la propiedad de ser hermoso y " $a$ " a Acautli, de manera que el hecho que la hace verdadera es el que Acautli, ese individuo designado por "Acautli" en el mundo real, tenga la propiedad $H$; ningún otro individuo que tuviese la propiedad $H$ en algún otro mundo posible $m_{n}$ haría verdadera la proposición en cuestión en $m_{n}$. En cambio, para que $p^{\prime}$ sea verdadera en algún otro mundo posible o situación contrafáctica, lo que se requiere es que el objeto que satisfaga la descripción "el primer azteca sacrificado en honor a Huitzilopochtli" en el mundo considerado, sea hermoso en ese mismo mundo. Es evidente que habrá un $m_{n}$ en el que Acautli no satisface la descripción definida en cuestión (por ejemplo, un mundo en el que Acautli no existe) sino que la satisface otro individuo, digamos, Tlacaélel; lo que haría verdadera a $p^{\prime}$ en ese mundo posible sería el hecho de que Tlacaélel, y no Acautli, fuese hermoso.

El intento de Kripke de disociar las categorías semánticas y metafísicas de todo aspecto epistemológico, parece llevarlo a lo siguiente: las oraciones singulares expresan proposiciones singulares, esto es, pares ordenados de la forma $<F^{n}\left\langle x_{1}, \ldots, x_{n}\right\rangle>$ en donde $F^{n}$ es una función proposicional $n$-ádica y $\left\langle x_{1}, \ldots, x_{n}\right\rangle$ es una secuencia n-ádica de objetos, tales que la proposición $F^{n}\left(x_{1} \ldots x_{n}\right)$ es verdadera, si y sólo si, la secuencia $n$-ádica $\left\langle x_{1} \ldots x_{n}\right\rangle$ satisface la función proposicional $F^{n}$. De esta manera, si representamos a Neptuno mediante " $a$ " y al predicado "ser la causa de las perturbaciones en la órbita de Urano" mediante " $F$ ", 
tenemos que la proposición expresada por "N es el $\varphi$ " es $\left\langle F^{1}<a>>\right.$. Esta proposición que representa un hecho en el mundo es igualado por Kripke al contenido cognoscitivo presente a la mente de Leverrier cuando asevera que $N$ es el $\varphi$. Kripke no distingue entre la proposición, que bien puede ser "lo expresado por una oración", y el contenido del pensamiento del hablante que utiliza la oración en cuestión.

La oración aseverada por Leverrier, esto es, " $\mathrm{N}$ es el $\varphi$ ", efectivamente expresa una proposición profundamente contingente, pero de ninguna manera es obvio que esa proposición constituya el contenido del pensamiento de Leverrier. ${ }^{17} \mathrm{Si} \mathrm{N}$ forma parte esencial de la proposición expresada por " $N$ es el $\varphi$ ", y Leverrier no sabe cuál es el objeto designado por " $N$ " difícilmente puede ese objeto formar parte del contenido de su pensamiento.

De acuerdo con lo anterior, tenemos que distinguir entre el hecho (o la proposición) que en una semántica formal se le asigna a una oración y el contenido cognoscitivo en el pensamiento de quien conoce lo expresado por la oración. ${ }^{18}$ Esta es la distinción que tanto Dummett como Evans enfatizan al sostener no sólo que $p$ y $p^{\prime}$ pueden estar asociadas a diferentes contenidos cognoscitivos aun cuando expresen la misma proposición, cosa que Frege ya había establecido, sino que a dos proposiciones diferentes (digamos, las expresadas por " $\mathrm{N}$ es

17 M. Dummett señala también esta cuestión cuando escribe: “un hecho comunicado por un enunciado, tal como lo entiende Kripke, no puede igualarse con su contenido cognoscitivo y... [esto] prepara la trampa en la que cae Kripke cuando habla de alguien que conoce a priori un hecho contingente». $O p$. cit., p. 126; véanse también las pp. 132 y ss. en donde Dummett discute la noción de significado en Kripke presentándola como una noción no epistemológica.

16 Para la distinción entre proposición y contenido cognoscitivo, véase esp. G. Evans, op. cit., pp. 176 y ss. y M. Dummett, op. cil., pp. 126 y ss. 
el $\varphi$ " y "el $\varphi$ es el $\varphi$ ") puede corresponderle un mismo contenido cognoscitivo en la mente de un sujeto. La solución que ofrece Evans al problema planteado por Kripke se apoya precisamente en esta distinción.

\section{La solución de Evans}

En su artículo "Reference and Contingency" Evans explora la posibilidad de contar en el lenguaje con nombres fregeanos, esto es, nombres que sí tienen un sentido, de manera que aun careciendo de referente puedan formar parte de oraciones significativas y, por ende, con condiciones de verdad. ${ }^{19}$ Dichos nombres descriptivos o fregeanos serían introducidos mediante una estipulación de la siguiente forma:

(E) Llamaremos "N" al individuo que sea el $\varphi$ en donde " $N$ " es un nombre y "el $\varphi$ " una descripción definida, la cual, para simplificar, ha de ser tenida como el único criterio para la aplicación del nombre descriptivo. Notemos que " $\mathrm{N}$ " sería un designador rígido, esto es, denotaría a un solo individuo en todo mundo posible, a saber, a aquel que de hecho, en el mundo real, satisface la descripción en cuestión. Es evidente que, en el ejemplo de Leverrier, la manera como éste introduce el nombre "Neptuno" se ajusta a lo anterior y por lo mismo es un nombre descriptivo en el sentido especificado por Evans.

Aceptar que hay nombres descriptivos no es lo mismo que aceptar simplemente que éstos sean abreviaturas de descripciones definidas. Evans admite de alguna manera la rigidez de los nombres así como la no rigidez de

19 Estos nombres, señala Evans, requerirían ser tratados mediante una "lógica libre" en la que se restringen las reglas de Generalización Existencial y de Especificación Universal, de manera que se impida pasar de premisas verdaderas a conclusiones falsas. 
las descripciones definidas, de manera que si un nombre descriptivo es efectivamente un nombre no podrá ser semánticamente equivalente a ninguna descripción. Evans sostiene, sin embargo, que el contenido en la mente de $S$ cuando usa una oración cuyo sujeto es un nombre descriptivo es idéntico al contenido en la mente de $S$ cuando sustituye en la oración el nombre descriptivo por la descripción mediante la cual introdujo el nombre. De manera que si Leverrier usa la descripción "la causa de las perturbaciones en la órbita de Urano" para introducir "Neptuno", al afirmar "Neptuno es $F$ " el contenido de su pensamiento es idéntico al contenido de su pensamiento cuando afirma "la causa de las perturbaciones en la órbita de Urano es $F^{\text {p }}$ aun cuando las proposiciones asociadas a las dos oraciones antes mencionadas sean diferentes en el sentido de ser diferenciables desde un punto de vista modal.

Ahora bien, si $F$ es la propiedad de ser la causa de las perturbaciones en la órbita de Urano, parecería seguirse de lo anterior que el contenido en la mente de Leverrier cuando afirma que Neptuno es $F$ es trivial o tautológico, pero sabemos que esto no es así, pues, como indicamos en la sección I, Leverrier pudo haber dicho consistentemente que Neptuno no es $F$. Evans evita la consecuencia anterior interpretando la descripción usada por Leverrier, con el fin de hacerla rígida, no como "la causa de las perturbaciones en la órbita de Urano", sino como "la causa de tales perturbaciones en el mundo real". De manera que el contenido en la mente de Leverrier cuando afirma que Neptuno es $F$, es que la causa de las perturbaciones en la órbita de Urano en $m^{*}$ es la causa de las perturbaciones en la órbita de Urano, y esto no es una situación necesaria, es contingente, pues es posible una situación contrafáctica o un mundo posible $m_{n}$ en el que la causa de las perturbaciones en la órbita de 
Urano en $m^{*}$ no sea la causa de las perturbaciones en la órbita de Urano en $m_{n}$. Lo que sucede con la afirmación de Leverrier, nos dice Evans, es que al ser hecha con respecto a $m^{*}$, se convierte en la afirmación de que la causa de las perturbaciones en la órbita de Urano en $m^{*}$ es la causa de las perturbaciones en la órbita de Urano en $m^{*}$. Y, como señala Evans, resulta poco sorprendente que Leverrier sepa a priori la verdad de su afirmación. ${ }^{20}$ Es evidente que Evans, quien acepta el análisis russelliano de las descripciones def inidas, no puede sostener que una oración existencialmente cuantificada pueda ser conocida a priori. Si queremos identificar con precisión lo que Leverrier conoce a priori, recordemos que "Neptuno" es un nombre descriptivo y que tales nombres requieren de una "lógica libre", esto es, una lógica que restrinja la aplicación de las reglas de Generalización Existencial y de Especificación Universal a dichos nombres. Evans sostiene, incluso, que el nombre "Neptuno" tiene que ser descriptivo (y tener alcance restringido) para que pueda surgir la paradoja, pues, si no lo fuera, la oración "Neptuno es el $\varphi$ " podría ser tratada conforme a una lógica clásica y estaría sujeta a la cuantificación existencial. ${ }^{21}$

¿Cómo interpretar, entonces, de manera más precisa, el contenido cognoscitivo de la oración afirmada por Leverrier? Evans piensa que lo conocido por Leverrier podría expresarse en una lógica clásica mediante una oración que tiene la forma de un condicional, a saber,

G. Evans sostiene que "podemos saber a priori que el mundo real posee ciertas propiedades que no poseen todos los mundos en tanto que la especificación de esas propiedades exija hacer referencia al mundo real o a lo que realmente es el caso". Op. cit., p. 183.

21 Si asi fuera, al conocer la verdad de " $N$ es el $\varphi$ " estaríamos en la posición de saber la verdad de la oración existencialmente cuantificada que resulta de aplicar la regla de G. E. a "N es el $\varphi$ "; pero obviamente nadie sostendría que la verdad de una oración cuantificada existencialmente pueda conocerse a priori. 
"Si existe la causa de las perturbaciones en la órbita de Urano, Neptuno es la causa de tales perturbaciones", en la cual "Neptuno" ocurre con alcance restringido, de manera que la oración podría simbolizarse de la siguiente manera:

$$
\text { 1. }(\exists x) \underline{\varphi(x)} \rightarrow[a \mid \dot{\varphi}(a)
$$

Esta oración está asociada a una proposición contingente, pues es posible una situación en la que el antecedente sea verdadero y el consecuente falso. La contingencia de 1 depende esencialmente del hecho de que "Neptuno" sea un nombre. Sin embargo, dada la manera como Leverrier fijó la referencia del nombre "Neptuno" el contenido de su pensamiento cuando afirma que Neptuno es el $\varphi$ parece ser equivalente a lo siguiente: ${ }^{22}$

$$
\text { 2. }(\exists x) \varphi(x) \rightarrow(\mathrm{I} x)\left(\varphi\left(x, m^{*}\right) ; \varphi(x)\right)
$$

La oración 2 es también contingente, pues, de nuevo, es posible que su antecedente sea verdadero y su consecuente falso. La propiedad que exige 2 a un mundo posible para hacerla verdadera es la siguiente:

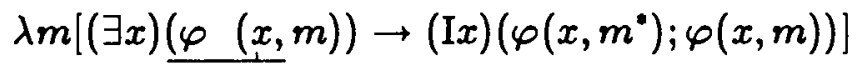

y esta es una propiedad, como señala Evans, que no todo mundo posible posee. ${ }^{23}$ Pero si aplicamos 2 al mundo real, como sucede con la afirmación de Leverrier, la oración 2 se convierte en

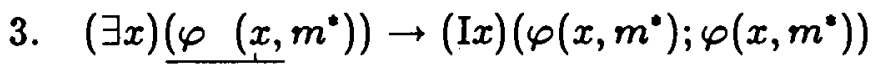

22 Sigo la notación utilizada por Evans, op. cil., p. 170, en donde introduce el cuantificador " $(\mathrm{I} x)^{n}$ y muestra cómo la oración " $(\mathrm{I} x)(\varphi(x)$; $\psi(x)$ " es demostrablemente equivalente a la expansión russelliana: $(\exists x)(\varphi(x) \& \psi(x))$.

23 Op. cit., p. 184. 
y esto es de acuerdo con Evans lo que Leverrier conoce a priori cuando afirma que Neptuno es la causa de las perturbaciones en la órbita de Urano. Como podemos observar, 3 resultará verdadera tanto en el caso en que algo sea el $\varphi$ en $m^{*}$ como en el caso en que nada sea el $\varphi$ en $m^{*}$, esto es, resultará verdadera en cualquier caso. Por eso no debe sorprendernos que Leverrier conozca a priori 'su verdad. La oración 2 es contingente en el sentido de que podría aplicarse a otros mundos posibles en los que resulta falsa, por ejemplo, un mundo posible $m_{n}$ en el que existe el $\varphi$ pero en el que Neptuno (esto es, el $\varphi$ en $m^{*}$ ) no es el $\varphi$ en $m_{n}$.

El carácter a priori de 3, nos dice Evans, depende del hecho de que en la especificación de la propiedad que se exige de un $m_{n}$ para la verdad de 2 se hace referencia al mundo real. Siempre que introduzcamos una propiedad en cuya especificación se haga referencia implícita o explícita al mundo real, a lo que realmente es el caso, podremos contar con oraciones que si bien están asociadas a proposiciones superficialmente contingentes pueden conocerse $a$ priori. 2 es contingente sólo en el sentido de que es posible su falsedad en algún otro mundo posible diferente de $m^{*}$, pero no es profundamente contingente, pues al aplicarla al mundo real está garantizada la existencia de una situación objetiva que la verifique. Al conocer a priori la verdad de 3 no aprendemos nada acerca del mundo real. La existencia de estas oraciones superficialmente contingentes y conocidas a priori, nos dice Evans, no tiene por qué inquietarnos.

La brillante argumentación de Evans nos deja, sin embargo, con otra inquietud. ¿Es adecuado interpretar la oración dicha por Leverrier como una especie de condicional encubierto? De ser así, bastaría con que el antecedente del condicional fuese falso, esto es, con que 
no existiera ninguna causa de las perturbaciones en la órbita de Urano, para que pudiéramos decir con verdad que Leverrier sabe que Neptuno es el $\varphi$. Esto resulta contraintuitivo: (i) Seguramente Leverrier hubiera protestado si alguien hubiera venido a decirle que lo que sabía realmente era la verdad de 3 . Su afirmación pretendió ser sustancial; de ninguna manera pensó hacer una afirmación que pudiera resultar vacuamente verdadera, menos aun conocida a priori; (ii) Leverrier sabía que había una causa de las perturbaciones en la órbita de Urano y esto lo sabía con base en sus observaciones y cálculos, esto es, lo sabía empíricamente; (iii) ¿qué sentido tiene entonces interpretar la afirmación de Leverrier como un condicional, si admitimos que conocía la verdad del antecedente? Leverrier parte de su conocimiento empírico de que existe el $\varphi$ para darle luego un nombre a un objeto que él sabe que existe aunque no sepa cuál es ese objeto. Por otra parte, es innegable que son poderosos los argumentos de Evans en favor de considerar que el contenido cognoscitivo de una oración en la que aparece como sujeto un nombre descriptivo es idéntico al contenido cognoscitivo de otra oración que resulta de sustituir dicho nombre descriptivo por la descripción mediante la que se introdujo el nombre. ¿Qué es, pues, lo que Leverrier sabe cuando sabe que $\mathrm{N}$ es el $\varphi$ ?

Parecería que lo que sabe Leverrier es algo como lo siguiente:

$$
\text { 4. }(\exists x) \varphi(x)
$$

y

$$
\text { 5. }(\mathrm{I} x)\left(\varphi\left(x, m^{\bullet}\right) ; \varphi(x)\right)
$$

esto es, parece más adecuado describir el contenido de su afirmación como una conjunción de 4 y 5 , y no como un condicional. 4, como hemos señalado, es algo que Le- 
verrier sabe empíricamente y su conocimiento de 5 depende a todas luces de su conocimiento de 4.5 tampoco puede conocerse $a$ priori, pues es también un enunciado existencial. Ambas son oraciones contingentes (profundamente contingentes) y ambas son conocidas a posteriori. Por lo tanto, no parece haber razón para exigir que el nombre "Neptuno" en la oración afirmada por Leverrier sea tratado con una "lógica libre" y que no esté abierto a la cuantificación existencial. Podemos aceptar con Evans que "Neptuno" en este contexto es un nombre fregeano, pero sólo en el sentido de tener asociado un contenido descriptivo, no en el sentido de no comprometernos con la existencia de un objeto en $m^{*}$. Si no existe Neptuno, Leverrier conoce sin lugar a dudas el significado de su afirmación, pero su oración no puede ser tenida como verdadera.

Resumiendo nuestro examen de la polémica entre Donnellan y Evans tenemos lo siguiente: (a) Donnellan parece equivocarse al sostener que Leverrier desconocía parcialmente el significado de la oración "Neptuno es el $\varphi^{\text {"; }}$ la distinción de Evans entre la proposición expresada por una oración y el contenido cognoscitivo en la mente del hablante que utiliza la oración, nos permite atribuirle a Leverrier un conocimiento cabal del significado de su oración. (b) También parece equivocarse Donnellan al sostener que Leverrier sabe a priori que "N es el $\varphi$ " expresa una verdad, pues para que Leverrier sepa esto es necesario que sepa que existe una y sólo una causa de las perturbaciones en la órbita de Urano y esto sólo lo puede saber empíricamente. Esto es, la única manera como Leverrier puede justificar su conocimiento de que " $\mathrm{N}$ es el $\varphi$ " expresa una verdad, es apelando a su conocimiento empírico de que existe el $\varphi$ en $m^{*}$ y recordando su propia estipulación. Si no sabe que existe el $\varphi$ en $m^{*}$, o si ha olvidado su propia 
estipulación, no puede saber, menos aun a priori, que la oración "N es el $\varphi$ " expresa una verdad. (c) El contenido cognoscitivo de la oración dicha por Leverrier es epistémicamente equivalente al contenido de la oración "El $\varphi$ en $m^{*}$ es el $\varphi$ ". No parece adecuado atribuirle a su afirmación la forma lógica de un condicional, como lo hace Evans, pues Leverrier sabe que es verdadero el antecedente de ese condicional. (d) Donnellan tiene razón al pensar que el nombre "Neptuno" en la frase de Leverrier debe estar abierto a la cuantificación existencial, pero no exactamente por las razones que él aduce, sino porque "Neptuno" tiene asociado el sentido de una descripción definida (y rígida). (e) El análisis adecuado

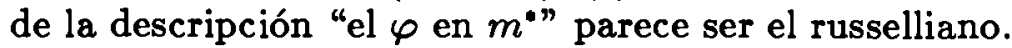
Esto es, para que la oración "el $\varphi$ en $m^{*}$ es el $\varphi^{\prime}$ " sea verdadera es necesario que exista algo en $m^{*}$ que sea el $\varphi$. Por lo tanto no parece haber lugar para atribuirle a Leverrier ningún conocimiento a priori cuando sabe que Neptuno es el $\varphi$.

¿Por qué Evans insiste en la falta de compromiso existencial cuando se conoce una oración contingente en la que aparece un nombre descriptivo? Creo que la respuesta es doble: (i) En primer lugar, Evans quiere tratar de la misma manera aquellos casos, como el de Leverrier, en los que sabemos que hay algo que satisface la descripción mediante la que introdujimos el nombre $\mathrm{y}$ otros casos en los que los nombres descriptivos pudieran ser introducidos mediante descripciones de las que ignoramos en absoluto si hay individuos que las satisfacen. Evans piensa que no es relevante para determinar "lo que conoce" un hablante que hace uso de una oración en la que aparece un nombre descriptivo el hecho de que sepa si la descripción mediante la que introdujo el nombre tiene o no un referente semántico. Así, por ejemplo, si yo estipulo: “Pepe' será el nombre 
del primer oso polar que pisa suelo congolés" y luego afirmo que Pepe es el primer oso polar que pisa suelo congolés, de acuerdo con Evans el conocimiento que tengo de lo que afirmo es a priori y del mismo tipo que el conocimiento que tiene Leverrier cuando sabe que Neptuno es la causa de las perturbaciones en la órbita de Urano. La ventaja que pueda haber en tratar semánticamente a "Neptuno" y "Pepe" de la misma manera, parece perderse cuando nos percatamos de sus consecuencias epistemológicas contraintuitivas. (ii) En segundo lugar, Evans asume correctamente que, salvo especificación en contrario, cuando usamos oraciones en las que aparecen nombres descriptivos, nuestra intención implícita es la de denotar al individuo que en el mundo real satisface la descripción, de manera que el sentido asociado a esos nombres es equivalente al de las descripciones introductoras subindexadas con " $m$ " ; pero, por otro lado, como nada nos garantiza que toda descripción de la forma "el $\varphi$ en $m^{*}$ tenga un referente semántico, nuestras pretendidas aserciones categóricas que hacen uso de tales descripciones no deben ser tratadas más que como modestos condicionales. Sin embargo, cuando sabemos que existe el referente de nuestra descripción introductora "el $\varphi$ en $m^{*}$ " de un nombre descriptivo $N$ (lo que, por otra parte, sucede en la mayoría de los casos) ¿qué caso tiene insistir en la falta de compromiso existencial de nuestra afirmación de la oración " $N$ es el $\varphi$ "?

Como habíamos señalado antes, hay dos maneras de entender la frase "lo que conoce $S$ cuando sabe que $\varphi x^{n}$. Donnellan y Kripke consideran que lo que conoce $S$ cuando sabe que $\varphi x$, es la proposición singular asociada a la oración $\varphi x$, y ésta es entendida como una entidad extramental, como el hecho de tener el individuo fijo denotado por $x$ la propiedad $\varphi$. Evans, en 
cambio, considera que lo relevante para determinar lo que conoce $S$ cuando sabe que $\varphi x$ es el contenido en la mente de $S$ cuando cree o acepta la oración en cuestión. El problema que se les presenta a los primeros es que, al identificar "lo que conoce" $S$ con la proposición asociada a la oración mediante la que expresa su creencia, suelen concederle al sujeto "más" conocimiento del que en realidad posee. El problema que se les presenta a quienes, como Evans, distinguen entre el contenido cognoscitivo y la proposición expresada, es que en ocasiones parecen atribuir al sujeto "menos" conocimiento del que en realidad tiene (o un conocimiento de otro tipo), como pudimos ver con el caso de Leverrier.

Si nuestro problema es determinar el contenido cognoscitivo de un sujeto $S$ que expresa su pretendido conocimiento mediante una oración singular en la que figura un nombre descriptivo (en el sentido especificado por Evans), parece crucial distinguir entre dos tipos de situaciones epistemológicas: (i) aquella en la que $S$ sabe que hay algo que satisface la descripción accidental mediante la que introdujo el nombre, y (ii) aquella en la que $S$ no sabe tal cosa. En el primer caso (que sería el de Leverrier), si decimos que $S$ sabe que $N$ es el $\varphi$, parece inevitable, como he argumentado, atribuir a $S$ un conocimiento empírico; en el segundo caso, resulta contrario a nuestras intuiciones atribuirle al sujeto cualquier tipo de conocimiento.

Lo anterior plantea la necesidad de revisar la noción kripkeana de "fijar la referencia" y distinguir entre los casos (i) y (ii) antes mencionados. Pero eso sería ya asunto de otro trabajo. 